\title{
A relatively short self-contained proof of the Baker-Campbell-Hausdorff theorem
}

\author{
Harald Hofstätter \\ Universität Wien, Institut für Mathematik, Oskar-Morgenstern-Platz 1, A-1090 Wien, \\ Austria
}

\begin{abstract}
We give a new purely algebraic proof of the Baker-Campbell-Hausdorff theorem, which states that the homogeneous components of the formal expansion of $\log \left(\mathrm{e}^{A} \mathrm{e}^{B}\right)$ are Lie polynomials. Our proof is based on a recurrence formula for these components and a lemma that states that if under certain conditions a commutator of a non-commuting variable and a given polynomial is a Lie polynomial, then the given polynomial itself is a Lie polynomial.
\end{abstract}

Keywords: Baker-Campbell-Hausdorff theorem, free Lie algebra, Lie polynomial

2010 MSC: 17B01

\section{Introduction}

Let $\mathbb{Q}\langle\langle\mathcal{A}\rangle\rangle$ denote the ring of formal power series with rational coefficients in non-commuting variables from a set $\mathcal{A} \sqrt{1}$ We assume that the set $\mathcal{A}$ is finite and contains at least two elements. In this ring exponential and logarithm functions are defined by their power series expansions

$$
\exp (X)=\mathrm{e}^{X}=\sum_{n=0}^{\infty} \frac{1}{n !} X^{n}, \quad \log (1+X)=\sum_{n=1}^{\infty} \frac{(-1)^{n+1}}{n} X^{n}
$$

for elements $X$ of $\mathbb{Q}\langle\langle\mathcal{A}\rangle\rangle$ without constant term. For two non-commuting variables $A, B$ we consider the formal expansion of

$$
C=\log \left(\mathrm{e}^{A} \mathrm{e}^{B}\right)=C_{1}+C_{2}+\ldots
$$

in $\mathbb{Q}\langle\langle A, B\rangle\rangle$, where for each $n \geq 1$ all terms of degree $n$ are collected in the homogeneous polynomial $C_{n}$ of degree $n$. The Baker-Campbell-Hausdorff (BCH)

\footnotetext{
Email address: hofi@harald-hofstaetter.at (Harald Hofstätter)

$U R L$ : www .harald-hof staetter.at (Harald Hofstätter)

${ }^{1}$ In the following we assume that the basic field is $\mathbb{Q}$, because this is the natural setting for computations around the Baker-Campbell-Hausdorff theorem. We note that everything remains valid if $\mathbb{Q}$ is consistently replaced by an extension field $K \supset \mathbb{Q}$ like $\mathbb{R}$ or $\mathbb{C}$.
} 
theorem states that each of these polynomials $C_{n}$ can be written as a Lie polynomial, i.e., a linear combination of $A$ and $B$ and (possibly nested) commutator terms in $A$ and $B$, see [3, Section 7.6].

The record for the shortest self-contained proof of the $\mathrm{BCH}$ theorem is presumably held by M. Eichler with his ingenious two-page proof [1], see also [3, Section 7.7]. This purely algebraic proof requires almost no theoretical preparation, but, on the other hand, it does not provide deeper insights into why the $\mathrm{BCH}$ theorem is true. In this last aspect, our own approach to a proof seems to be more illuminating.

\section{Towards a proof of the $\mathrm{BCH}$ theorem}

We introduce some notations. Let $\mathbb{Q}\langle\mathcal{A}\rangle \subset \mathbb{Q}\langle\langle\mathcal{A}\rangle\rangle$ denote the subring of all formal power series with only finitely many non-zero coefficients, i.e., the ring of polynomials in the non-commuting variables $\mathcal{A}$. $\mathbb{Q}\langle\mathcal{A}\rangle$ considered as a vector space over the field $\mathbb{Q}$ with Lie bracket defined by the commutator $[X, Y]=X Y-Y X$ is a Lie algebra, i.e., the Lie bracket is bilinear and satisfies

$$
\begin{array}{lc}
{[X, Y]=-[Y, X]} & \text { (anti-symmetry) } \\
{[X,[Y, Z]]+[Z,[X, Y]]+[Y,[Z, X]]=0 \quad \text { (Jacobi identity) }}
\end{array}
$$

for all $X, Y, Z \in \mathbb{Q}\langle\mathcal{A}\rangle$, see $\left[3\right.$, Section 4.4]. We define $\mathcal{L}_{\mathbb{Q}}(\mathcal{A}) \subset \mathbb{Q}\langle\mathcal{A}\rangle$ as the smallest subspace of $\mathbb{Q}\langle\mathcal{A}\rangle$ containing $\mathcal{A}$ and being closed under Lie brackets. $\mathcal{L}_{\mathbb{Q}}(\mathcal{A})$ is called the free Lie algebra generated by $\mathcal{A}$. It is clear that the elements of $\mathcal{L}_{\mathbb{Q}}(\mathcal{A})$ are precisely the linear combinations of variables from $\mathcal{A}$ and simple and nested Lie brackets in these variables, i.e., the Lie polynomials in the variables $\mathcal{A}$.

We begin our considerations aiming at a proof of the $\mathrm{BCH}$ theorem with the well-known formal identity

$$
\mathrm{e}^{X} Y \mathrm{e}^{-X}=\mathrm{e}^{\operatorname{ad} X}(Y):=\sum_{n=0}^{\infty} \frac{1}{n !} \operatorname{ad}_{X}^{n}(Y) .
$$

Here $\operatorname{ad}_{X}$ is the linear operator defined by $Y \mapsto[X, Y]=X Y-Y X$ such that $\operatorname{ad}_{X}^{n}(Y)=[X,[X,[\ldots[X, Y] \ldots]]$ with $X$ occurring $n$ times. The proof of (2) is easy: We define linear operators $L_{X}, R_{X}$ by $L_{X}(Z)=X Z, R_{X}(Z)=Z X$ such that $\operatorname{ad}_{X}=L_{X}-R_{X}$. These operators commute, because $L_{X} R_{X}(Z)=$ $X Z X=R_{X} L_{X}(Z)$. It follows

$$
\mathrm{e}^{\operatorname{ad}_{X}}(Y)=\mathrm{e}^{L_{X}-R_{X}}(Y)=\mathrm{e}^{L_{X}} \mathrm{e}^{-R_{X}}(Y)=\mathrm{e}^{L_{X}} \mathrm{e}^{R_{-X}}(Y)=\mathrm{e}^{X} Y \mathrm{e}^{-X} .
$$

Using $\mathrm{e}^{C}=\mathrm{e}^{A} \mathrm{e}^{B}$ with $C$ defined by (11) and applying (2) twice we obtain

$$
\mathrm{e}^{\operatorname{ad}_{C}}(B) \mathrm{e}^{C}=\mathrm{e}^{C} B=\mathrm{e}^{A} \mathrm{e}^{B} B=\mathrm{e}^{A} B \mathrm{e}^{B}=\mathrm{e}^{\operatorname{ad}_{A}}(B) \mathrm{e}^{C},
$$

and thus, after cancelling the factor $\mathrm{e}^{C}$,

$$
\sum_{k=0}^{\infty} \frac{1}{k !} \operatorname{ad}_{C}^{k}(B)=\sum_{k=0}^{\infty} \frac{1}{k !} \operatorname{ad}_{C_{1}+C_{2}+\ldots}^{k}(B)=\sum_{k=0}^{\infty} \frac{1}{k !} \operatorname{ad}_{A}^{k}(B) .
$$


In this equation consider the terms of degree $n+1$, which leads to

$$
\sum_{m=1}^{n} \frac{1}{m !} \sum_{\substack{k_{1}+\cdots+k_{m}=n \\ k_{j} \geq 1}} \operatorname{ad}_{C_{k_{1}}} \circ \operatorname{ad}_{C_{k_{2}}} \circ \cdots \circ \operatorname{ad}_{C_{k_{m}}}(B)=\frac{1}{n !} \operatorname{ad}_{A}^{n}(B),
$$

or, after splitting off the term for $m=1$ from the sum and reordering,

$$
\left[B, C_{n}\right]=\sum_{m=2}^{n} \frac{1}{m !} \sum_{\substack{k_{1}+\cdots+k_{m}=n \\ k_{j} \geq 1}} \operatorname{ad}_{C_{k_{1}}} \circ \operatorname{ad}_{C_{k_{2}}} \circ \cdots \circ \operatorname{ad}_{C_{k_{m}}}(B)-\frac{1}{n !} \operatorname{ad}_{A}^{n}(B) .
$$

Here we make two observations:

1. Provided equation (3) can be uniquely solved for $C_{n}$, it can be used as a recurrence formula for the computation of the $C_{n}$.

2. If we assume that all $C_{k}$ for $k<n$ are Lie polynomials in $A, B$, then it is obvious that the right-hand side of (3) is also a Lie polynomial. If we can show that $C_{n}$ is a Lie polynomial provided $\left[B, C_{n}\right]$ is a Lie polynomial, then, using induction on $n$, this leads to a proof of the $\mathrm{BCH}$ theorem.

With regard to the first observation, the following lemma gives a criterion for the uniqueness of a solution of (3). (Because $C_{n}$ defined by (11) is a solution, there is no question about existence.)

Lemma 1. If $\operatorname{ad}_{a}(P)=[a, P]=0$ for $a \in \mathcal{A}$ and $P \in \mathbb{Q}\langle\mathcal{A}\rangle$ not containing any term $\alpha_{k} a^{k}, \alpha_{k} \neq 0, k \geq 0$, then $P=0$.

Proof. Towards a contradiction, we assume that there exists a word $w=$ $w_{1} \cdots w_{n}, w_{j} \in \mathcal{A}$ with non-zero coefficient $(P, w)$ in the expansion $P=$ $\sum_{w}(P, w) w$. $w$ must contain at least one letter $w_{j} \neq a$ and thus has the form $w=v x a^{k}$ with $k \geq 0$ for some word $v$ and $x \in \mathcal{A}, x \neq a$. Let $\widetilde{w}$ be a word with $(P, \widetilde{w}) \neq 0$ of this form with $k$ maximal. Then the coefficient $\left(\operatorname{ad}_{a}(P), \widetilde{w} a\right)$ of $\widetilde{w} a$ in $\operatorname{ad}_{a}(P)=\sum_{w}(P, w)(a w-w a)$ is $\left(\operatorname{ad}_{a}(P), \widetilde{w} a\right)=-(P, \widetilde{w})$ because $a w \neq \widetilde{w} a$ for all words $w$ by the maximality of $\widetilde{w}$. Because $(P, \widetilde{w}) \neq 0$ this is a contradiction to $\operatorname{ad}_{a}(P)=0$.

We have thus established the uniqueness of the solution of (3) up to a term $\beta_{n} B^{n}$. By the following lemma no such term can actually occur if $n \geq 2$. Therefore, for $n \geq 2$ the solution of (3) is indeed unique. Furthermore, the lemma gives us the initial value $C_{1}=A+B$ for the recursion.

Lemma 2. In the expansion of $C=\log \left(\mathrm{e}^{A} \mathrm{e}^{B}\right)=A+B+[$ terms of degrees $\geq 2]$ in $\mathbb{Q}\langle\langle A, B\rangle\rangle$ there are no terms $\alpha_{k} A^{k}, \alpha_{k} \neq 0, k \geq 2$ or $\beta_{k} B^{k}, \beta_{k} \neq 0, k \geq 2$.

Proof. Write $\mathrm{e}^{A} \mathrm{e}^{B}=e^{B}+h(A, B)$ where each term in the expansion of $h(A, B)=\left(e^{A}-1\right) e^{B}$ contains at least one $A$. Then

$$
\begin{aligned}
C & =\log \mathrm{e}^{A} \mathrm{e}^{B}=\sum_{n=1}^{\infty} \frac{(-1)^{n+1}}{n}\left(\left(e^{B}-1\right)+h(A, B)\right)^{n} \\
& =\sum_{n=1}^{\infty} \frac{(-1)^{n+1}}{n}\left(e^{B}-1\right)^{n}+H(A, B)=\log \left(\mathrm{e}^{B}\right)+H(A, B)=B+H(A, B),
\end{aligned}
$$


where each term in the expansion of $H(A, B)$ contains at least one $A$. A similar argument leads to $C=\log \mathrm{e}^{A} \mathrm{e}^{B}=A+\widetilde{H}(A, B)$, where each term in the expansion of $\widetilde{H}(A, B)$ contains at least one $B$.

With regard to the second of the above observations, it follows from Lemma2 and Lemma 3 below that for $n \geq 2, C_{n}$ indeed is a Lie polynomial provided $\left[B, C_{n}\right]$ is a Lie polynomial. As already mentioned, this together with $C_{1}=$ $A+B$ completes the proof of the $\mathrm{BCH}$ theorem.

\section{A lemma crucial for our proof of the $\mathrm{BCH}$ theorem}

Lemma 3. Let $a \in \mathcal{A}$ and $P \in \mathbb{Q}\langle\mathcal{A}\rangle$ not containing any term $\alpha_{k} a^{k}, \alpha_{k} \neq 0$, $k \geq 0$. If $\operatorname{ad}_{a}(P)=[a, P]$ is a Lie polynomial, then $P$ is a Lie polynomial.

It is possible to give a short (but not self-contained) proof of this lemma by taking strong results from the theory of free Lie algebras like [2, Theorem 1.4] for granted. The following self-contained proof was essentially obtained by distilling from [2, Chapter 1] just enough material to derive Lemma 3,

Our proof is based on properties of the right normed bracketing which for words $w=w_{1} \cdots w_{n}, w_{j} \in \mathcal{A}$ is defined by $r(w)=\left[w_{1},\left[w_{2},\left[\ldots\left[w_{n-1}, w_{n}\right] \ldots\right]\right]\right]$. Because the set of words is a basis of the vector space $\mathbb{Q}\langle\mathcal{A}\rangle, r$ can uniquely be extended to a linear map $r: \mathbb{Q}\langle\mathcal{A}\rangle \rightarrow \mathbb{Q}\langle\mathcal{A}\rangle$ satisfying the basic identities

$$
r(x)=x, \quad r(x P)=\operatorname{ad}_{x}(r(P)), \quad x \in \mathcal{A}, P \in \mathbb{Q}\langle\mathcal{A}\rangle .
$$

By recursive applications of the Jacobi identity written as

$$
[[X, Y], Z]=[X,[Y, Z]]-[Y,[X, Z]]
$$

to a Lie polynomial $P \in \mathcal{L}_{\mathbb{Q}}(\mathcal{A})$, we eventually obtain a linear combination of right normed elements $\left[a_{1},\left[a_{2},\left[\ldots\left[a_{n-1}, a_{n}\right] \ldots\right]\right]\right], n \geq 1, a_{j} \in \mathcal{A}$. This shows that for each Lie polynomial $P \in \mathcal{L}_{\mathbb{Q}}(\mathcal{A})$ there exists a polynomial $\widetilde{P} \in \mathbb{Q}\langle\mathcal{A}\rangle$ such that $P=r(\widetilde{P})$.

After the following sequence of Lemmas 46 based on [2, Section 1.6.6] and an auxiliary result from Lemma 7 , the proof of Lemma 3 will finally succeed in a relatively simple way.

Lemma 4. For polynomials $P, Q \in \mathbb{Q}\langle\mathcal{A}\rangle$ we have

$$
r(r(P) Q)=[r(P), r(Q)] .
$$

Proof. Because both sides of the identity are linear in $P$, we only have to consider the special case where $P$ is a word. We use induction on the length $n$ of $P$. For $n=1$ we have $P \in \mathcal{A}$ and (4) follows immediately. For $n>1$ write 
$P=x \widetilde{P}$ with $x \in \mathcal{A}$ and $\widetilde{P}$ a word of length $n-1$. Then,

$$
\begin{aligned}
& r(r(P) Q)=r(r(x \widetilde{P}) Q)=r([x, r(\widetilde{P})] Q)=r(x r(\widetilde{P}) Q)-r(r(\widetilde{P}) x Q) \\
& \quad=[x, r(r(\widetilde{P}) Q)]-[r(\widetilde{P}), r(x Q)] \text { (2nd term by induction hypothesis) } \\
& \quad=[x,[r(\widetilde{P}), r(Q)]]-[r(\widetilde{P}),[x, r(Q)]] \text { (1st term by induction hypothesis) } \\
& \quad=[[x, r(\widetilde{P})], r(Q)] \text { (by Jacobi identity) } \\
& \quad=[r(P), r(Q)] .
\end{aligned}
$$

Lemma 5. For Lie polynomials $P_{1}, P_{2} \in \mathcal{L}_{\mathbb{Q}}(\mathcal{A})$ we have

$$
r\left(\left[P_{1}, P_{2}\right]\right)=\left[P_{1}, r\left(P_{2}\right)\right]+\left[r\left(P_{1}\right), P_{2}\right] .
$$

Proof. There exist polynomials $\widetilde{P}_{1}, \widetilde{P}_{1}$ such that $P_{1}=r\left(\widetilde{P}_{1}\right), P_{2}=r\left(\widetilde{P}_{2}\right)$. From Lemma 4 it follows

$$
\begin{aligned}
& r\left(\left[P_{1}, P_{2}\right]\right)=r\left(\left[r\left(\widetilde{P}_{1}\right), r\left(\widetilde{P}_{2}\right)\right]\right)=r\left(r\left(\widetilde{P}_{1}\right) r\left(\widetilde{P}_{2}\right)\right)-r\left(r\left(\widetilde{P}_{2}\right) r\left(\widetilde{P}_{1}\right)\right) \\
& \quad=\left[r\left(\widetilde{P}_{1}\right), r^{2}\left(\widetilde{P}_{2}\right)\right]-\left[r\left(\widetilde{P}_{2}\right), r^{2}\left(\widetilde{P}_{1}\right)\right]=\left[r\left(\widetilde{P}_{1}\right), r^{2}\left(\widetilde{P}_{2}\right)\right]+\left[r^{2}\left(\widetilde{P}_{1}\right), r\left(P_{2}\right)\right] \\
& \quad=\left[P_{1}, r\left(P_{2}\right)\right]+\left[r\left(P_{1}\right), P_{2}\right] .
\end{aligned}
$$

Lemma 6. If $P \in \mathcal{L}_{\mathbb{Q}}(\mathcal{A})$ is a homogeneous Lie polynomial of degree $n$, then

$$
r(P)=n P .
$$

Proof. We use induction on $n$. For $n=1, P=\sum_{a \in \mathcal{A}} \alpha_{a} a$ and (5) follows immediately. If $n>1$, then $P$ is a linear combination of elements of the form $\left[P_{1}, P_{2}\right]$ where $P_{1}, P_{2}$ are homogeneous Lie polynomials of degrees $n_{1}, n_{2} \geq 1$ with $n_{1}+n_{2}=n$. For such elements we have

$$
r\left(\left[P_{1}, P_{2}\right]\right)=\left[r\left(P_{1}\right), P_{2}\right]+\left[P_{1}, r\left(P_{2}\right)\right]=n_{1}\left[P_{1}, P_{2}\right]+n_{2}\left[P_{1}, P_{2}\right]=n\left[P_{1}, P_{2}\right],
$$

where we used Lemma 5 and the induction hypothesis $r\left(P_{1}\right)=n_{1} P_{1}, r\left(P_{2}\right)=$ $n_{2} P_{2}$ for $n_{1}, n_{2}<n$. Using linearity of $r$ we obtain (5).

Lemma 7. If $a \in \mathcal{A}$ and $P \in \mathcal{L}_{\mathbb{Q}}(\mathcal{A})$ is a Lie polynomial, then

$$
r(P a)=-\operatorname{ad}_{a}(P) .
$$

Proof. By linearity, it suffices to consider the case where $P$ is homogeneous of degree $n \geq 1$. Using Lemma 6 we obtain

$$
\begin{aligned}
r(P a) & =r(a P)-r\left(\operatorname{ad}_{a}(P)\right)=\operatorname{ad}_{a}(r(P))-r\left(\operatorname{ad}_{a}(P)\right) \\
& =n \operatorname{ad}_{a}(P)-(n+1) \operatorname{ad}_{a}(P)=-\operatorname{ad}_{a}(P) .
\end{aligned}
$$

Proof of Lemma 3 Let $a \in \mathcal{A}$ and $P \in \mathbb{Q}\langle\mathcal{A}\rangle$ not containing any term $\alpha_{k} a^{k}, \alpha_{k} \neq 0, k \geq 0$ such that $\operatorname{ad}_{a}(P)$ is a Lie polynomial. We want to show that $P$ is a Lie polynomial. Splitting $P$ into homogeneous components and $\operatorname{ad}_{a}(P)$ into corresponding homogeneous components, it suffices to consider the 
case where $P$ is a homogeneous polynomial of degree $n$ without a term $\alpha_{n} a^{n}$ and $\operatorname{ad}_{a}(P)$ is a homogeneous Lie polynomial of degree $n+1$. From Lemma 7 it follows

$$
-\operatorname{ad}_{a}^{2}(P)=r\left(\operatorname{ad}_{a}(P) a\right)=r(a P a)-\underbrace{r(P a a)}_{=0}=\operatorname{ad}_{a}(r(P a))
$$

and thus $\operatorname{ad}_{a}\left(\operatorname{ad}_{a}(P)+r(P a)\right)=0$ such that

$$
\operatorname{ad}_{a}(P)=-r(P a)
$$

by Lemma 12 Using Lemma 6 we obtain

$$
(n+1) \operatorname{ad}_{a}(P)=r\left(\operatorname{ad}_{a}(P)\right)=r(a P)-r(P a)=\operatorname{ad}_{a}(r(P))+\operatorname{ad}_{a}(P)
$$

and thus $n \operatorname{ad}_{a}(P)=\operatorname{ad}_{a}(r(P))$ from which it follows

$$
\operatorname{ad}_{a}\left(P-\frac{1}{n} r(P)\right)=0 .
$$

Using Lemma 1 we obtain $P=\frac{1}{n} r(P)$ where the right-hand side is obviously a Lie polynomial.

\section{References}

[1] M. Eichler. A new proof of the Baker-Campbell-Hausdorff formula. J. Math. Soc. Japan, 20 (1968) 23-25.

[2] C. Reutenauer. Free Lie Algebras. LMS monographs. Oxford Univ. Press, 1993.

[3] J. Stillwell. Naive Lie Theory. Undergraduate Texts in Mathematics. Springer New York, 2010.

\footnotetext{
${ }^{2}$ Note that for the last equation we cannot use Lemma 7 directly, because we do not (yet) know that $P$ is a Lie polynomial.
} 